\title{
A THEOREM ON NEAR EQUICONTINUITY OF TRANSFORMATION GROUPS
}

\author{
FRED A. ROBERSON
}

ABSTRACT. A transformation group is nearly equicontinuous if the set of nonequicontinuous points is zero dimensional and compact. It has been shown that if a transformation group is nearly equicontinuous with locally compact, locally connected metric phase space and if the set of equicontinuous points is connected, then the set $N$ of nonequicontinuous points can contain at most two minimal sets. In this paper we will show that if in addition the phase space is not compact, then $N$ contains exactly one minimal set.

The purpose of this paper is to prove an additional theorem related to results in [3]. This theorem shows what happens when the phase space of [3] is assumed to be not compact. This result, in particular Corollary 3, is related to the results in [5] and [6]. Notation and definitions are as in the book [2]. All topological spaces considered are assumed to be Hausdorff.

THEOREM. Let $(X, T, \pi)$ be a transformation group such that $X$ is a locally compact, locally connected metric space which is not compact, the set $E$ of all points of $X$ at which $T$ is equicontinuous is connected, and the set $N=X-E$ is zero dimensional and compact. Then $N$ contains exactly one minimal set (that is, a nonempty set which is the orbit closure of each of its points).

This theorem will be proved by applying the theorem in [3] to the one-point compactification of $X$. The following remarks, which are known for the most part, show that the hypothesis of the theorem in [3] are satisfied.

REMARK 1. Let $(X, T, \pi)$ be a transformation group where $X$ is locally compact. Then $(\tilde{X}, T, \tilde{\pi})$ is a transformation group where $\tilde{X}$ is the one-point compactification of $X$ and $(\tilde{x}, t) \tilde{\pi}=\tilde{x}$ for each $t$ in $T$ and $(x, t) \tilde{\pi}=(x, t) \pi$ for each $x$ in $X$ and $t$ in $T$. Here $\tilde{x}$ is the one point added to make $X$ compact.

Proof. The first two axioms are trivial. That $\tilde{\pi}$ is continuous follows from 1.18 (4) of [2].

Received by the editors October 20, 1969.

AMS 1969 subject classifications. Primary 5482, 5480.

Key words and phrases. Metric space, transformation group, equicontinuity. 
Remark 2. Under the assumption of the theorem $X$ and $\tilde{X}$ are connected.

Proof. $E$ is dense in $X$ and connected and the result follows.

REMARK 3. Under the assumption of the theorem $\tilde{X}$ is metrizable.

Proof. This follows from the fact that $X$ is connected and the following theorems [1, Theorems XI.7.3, XI.7.2, IX.5.6, XI.8.6].

REMARK 4. If $(X, T, \pi)$ is a transformation group, if $U$ and $V$ are compatible uniformities of $X$, if $x \in X$ and if $\operatorname{cl}(x T)$ is compact, then $x$ is equicontinuous with respect to $U$ if and only if $x$ is equicontinuous with respect to $V$.

Proof. Use the Lesbesgue covering lemma for a compact subset of a uniform space.

REMARK 5. If $X$ is a locally compact space, then the uniformity of its one-point compactification $\tilde{X}$ induces on $X$ the least uniformity compatible with the topology of $X$.

REMARK 6. Let $(X, T, \pi)$ be a transformation group where $X$ is locally compact. Let $\tilde{E}$ be the set of equicontinuous points of $\widetilde{X}$ and $\tilde{N}=\tilde{X}-\tilde{E}$. If $N$ is compact, then $N \subset \tilde{N}$ and $E \subset \tilde{E}$.

Proof. The first statement follows from Remark 4 and the second from Remark 5.

REMARK 7. If $X$ is locally compact, connected and locally connected, then $\tilde{X}$ is locally connected.

Proof. This follows from [4, Theorem 3.9].

REMARK 8. Under the assumption of the theorem $\tilde{x}$ is in $\tilde{N}$.

Proof. This follows from property (4) on p. 62 of [3] (which shows that no equicontinuous point can be fixed).

It is easy to see from the above remarks that $(\tilde{X}, T, \tilde{\pi})$ satisfies the conditions of the theorem in [3], and that $\{\tilde{x}\}$ is minimal in $\tilde{N}$. Therefore $N$ can contain at most one minimal set in order that $\tilde{N}$ will contain at most two. But [2, Theorem 2.22] implies $N$ must contain at least one minimal set. Therefore, $N$ contains exactly one minimal set.

In the following corollaries the hypothesis of the theorem is assumed.

Corollary 1. If $x_{1}$ and $x_{2}$ belong to $X$, then $\operatorname{cl}\left(x_{1} T\right) \cap \mathrm{cl}\left(x_{1} T\right)$ is not empty.

Proof. If $x \in E$, then we know from [3] that $\operatorname{cl}(x T) \supset N$. If $x \in N$, then we know from [2, Theorem 2.22] that $\operatorname{cl}(x T)$ contains a minimal set. In either case $\operatorname{cl}(x T)$ must contain the minimal set of $N$.

Corollary 2. If $T$ is almost periodic at each point of $N$, then $N$ is minimal. 
COROLlaRy 3. If $T$ is abelian or connected, then $N$ contains exactly one point and this point is fixed under $T$.

Corollaries 2 and 3 follow in the same way as Corollaries 2 and 3 of [3].

\section{REFERENCES}

1. J. Dugundji, Topology, Allyn and Bacon, Boston, Mass., 1966. MR 33 \#1824.

2. W. H. Gottschalk and G. A Hedlund, Topological dynamics, Amer. Math. Soc. Colloq. Publ., vol. 36, Amer. Math. Soc., Providence, R. I., 1955. MR 17, 650.

3. W. J. Gray and F. A. Roberson, On the near equicontinuity of transformation groups, Proc. Amer. Math. Soc. 23 (1969), 59-63.

4. J. G. Hocking and G. S. Young, Topology, Addison-Wesley, Reading, Mass., 1961. MR 23 \#A2857.

5. T. Homma and S. Kinoshita, On homeomorphisms which are regular except for a finite number of points, Osaka Math. J. 7 (1955), 29-38. MR 16, 1140.

6. S. K. Kaul, On almost regular homeomorphisms, Canad. J. Math. 20 (1968), 1-6. MR 36 \#5908.

Florida State University, Tallahassee, Florida 32306 